\title{
INFLUÊNCIA DA IDADE E DA POSIÇÃO AO LONGO DO TRONCO NA COMPOSIÇÃO QUÍMICA DA MADEIRA DE Eucalyptus grandis Hill ex. Maiden ${ }^{1}$
}

José de Castro Silva ${ }^{2}$, Jorge Luis Monteiro de Matos $^{3}$, José Tarcísio da Silva Oliveira ${ }^{4}$ e Wesclei Viana Evangelista ${ }^{5}$

\begin{abstract}
RESUMO - Este trabalho estudou a variação da composição química na madeira de Eucalyptus grandis de quatro diferentes idades (10, 14, 20 e 25 anos), proveniente de talhões comerciais. As amostras foram coletadas de três discos, retirados da base e das extremidades das duas primeiras toras, de $3 \mathrm{~m}$ cada uma, de 16 árvores (quatro para cada idade), totalizando 48 discos. Os valores médios dos teores de holocelulose, lignina e extrativos foram de 69, 27 e 4\%, respectivamente. Verificou-se que os teores de extrativos e lignina aumentaram com a idade, com maiores concentrações nos discos próximos da base; verificou-se, também, que o teor de holocelulose diminuiu com a idade, com maiores concentrações nos discos retirados nas regiões superiores do tronco.
\end{abstract}

Palavras-chave: Eucalyptus grandis, composição química, idade e variação longitudinal.

\section{INFLUENCE OF AGE AND POSITION ALONG THE TRUNK ON THE CHEMICAL COMPOSITION OF Eucalyptus grandis Hill ex. Maiden WOOD}

\begin{abstract}
The objective of this work was to study the chemical composition variation of the Eucalyptus grandis wood, of four different ages (10,14,20 and 25 years), from commercial stands. The samples were removed from three disks taken from the base and top of the first two $3 \mathrm{~m} l o g s$, from sixteen trees (four per age), totalizing fourty-eight discs. The mean values of holocellulose, lignin and extractive contents were 69, 27 and 4\%, respectively. The extractive and lignin contents increased with age, with greater concentrations near the base; the holocellulose content also decreased with age with greater concentrations in discs removed from the upper parts of the trunk.
\end{abstract}

Key words: Eucalyptus grandis, chemical composition, age, longitudinal variation.

\section{INTRODUÇÃO}

A madeira é um material orgânico, e os seus constituintes químicos estão diretamente relacionados com as suas propriedades. A madeira, segundo Lepage (1986), é um biopolímero tridimensional, composto, principalmente, de celulose, hemiceluloses e lignina, responsáveis pela formação da parede celular e pela maioria de suas propriedades. Os extrativos, também de reconhecida importância em várias situações, atuam como componentes complementares e apresentam grande variabilidade em sua quantidade e constituição. $\mathrm{O}$ conhecimento da natureza química da madeira possibilita o entendimento de seu comportamento como matériaprima para diversos usos.

Segundo Hillis e Brown (1978), as diversas espécies de eucalipto apresentam a seguinte faixa de composição

\footnotetext{
${ }^{1}$ Recebido em 19.03.2003 e aceito para publicação em 20.04.2005.

${ }^{2}$ Departamento de Engenharia Florestal da UFV - 36570-000 Viçosa-MG.

${ }^{3}$ DETR/UFPR, Rua Lothário Meissner, 3.400, Jardim Botânico, 80210-170 Curitiba-PR.

${ }^{4}$ Departamento de Engenharia Rural da UFES, Caixa Postal 16, 29500-000 Alegre-ES.

${ }^{5}$ Programa de Pós-Graduação em Ciência Florestal da UFV, Viçosa-MG.
} 
química de sua sua madeira: $40-62 \%$ de celulose, 12 $22 \%$ de hemiceluloses e $15-25 \%$ de lignina. Segundo Raymond (2000), os teores de holocelulose e de extrativos aumentam com a idade, ocorrendo o inverso quanto aos teores de lignina. Diversos outros autores verificaram o inverso, quando os teores de lignina e extrativos apresentaram correlação direta com a idade e inversa com o teor de holocelulose.

Segundo Hillis e Brown (1978), Panshin e De Zeeuw (1980), o teor de extrativos é um dos mais importantes indicadores de conformidade da madeira para diversos usos industriais. Kramer e Koslowski (1979), Jankowsky (1979), Zobel e Bujtenen (1989), Gonçalez (1993) e Chafe (1994) afirmaram que, durante a formação do cerne, ampla variedade de substâncias extrativas, incluindo taninos, corantes, óleos, gomas, resinas e sais de ácidos orgânicos, acumula-se nos lumes das células e paredes celulares, resultando, às vezes, na coloração mais escura da madeira, além de aumento da massa específica e durabilidade.

Santos (1996) e Raymond (2000) afirmaram que as variações químicas dos componentes fundamentais da madeira não têm qualquer correlação com os índices de qualidade para produtos sólidos; as variações dos componentes secundários, ao contrário, podem interferir nas propriedades de usinagem e acabamento, exigindo, às vezes, ajustes de processos.

Este trabalho teve como objetivo avaliar a variabilidade da composição química da madeira de Eucalyptus grandis, de quatro diferentes idades (10, 14,20 e 25 anos) e três diferentes posições longitudinais, no sentido base-topo.

\section{MATERIAL E MÉTODOS}

O material utilizado neste estudo foi obtido de plantios comerciais de Eucalyptus grandis Hill ex. Maiden, com idades de 10, 14, 20 e 25 anos, procedentes da Fazenda Monte Alegre, da KLABIN Fabricadora de Papel e Celulose S. A., localizada no município de Telêmaco Borba, no Estado do Paraná.

A amostragem do material na árvore foi feita através da coleta de três discos, retirados da base e das extremidades das duas primeiras toras, ambas com o comprimento comercial de $3 \mathrm{~m}$, de cada uma das 16 árvores (quatro para cada idade), totalizando 48 discos. De cada disco, retirou-se uma cunha, correspondendo aproximadamente a $1 / 8$ da seção do disco, sendo, posteriormente, o material convertido em cavacos. Utilizando-se o moinho laboratorial Wiley, os cavacos foram transformados em serragem.

O material empregado nas análises químicas foi aquele que passou pela peneira de malha de 40 mesh e ficou retido na malha de 60 mesh, sendo utilizadas quatro repetições para cada amostra. $\mathrm{O}$ delineamento experimental foi o inteiramente casualizado, procedendose à análise estatística, através da análise de variância e do teste de médias (Tukey), considerando os efeitos da variação da idade e da posição ao longo do tronco, bem como a interação entre os efeitos idade x posição. As análises químicas e as respectivas normas adotadas foram as seguintes: extrativos totais (norma TAPPI T 624 om-82); lignina insolúvel (GOMIDE e DEMUNER, 1986); lignina solúvel (GOLDSCHIMID, 1971) e holocelulose (obtida por diferença).

O esquema de retirada das amostras está ilustrado na Figura 1.

\section{RESULTADOS E DISCUSSÃO}

\section{1. Teor de extrativos totais}

Pela análise de variância, verificou-se que os efeitos da idade e da posição do disco ao longo da árvore no teor de extrativos foram significativos a $95 \%$ de probabilidade. Foi verificado, ainda, que os efeitos da posição do disco ao longo do tronco foram muito mais pronunciados que a idade; e a interação desses

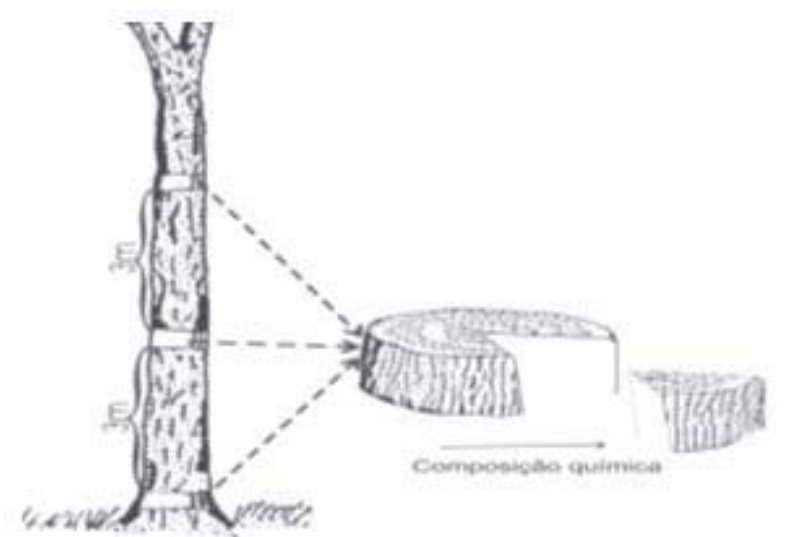

Figura 1 - Retirada das amostras para ensaios de composição química.

Figure 1 - Layout of chemical analysis sample location within the tree trunk. 
efeitos teve muito pouca influência na definição dos valores relacionados aos teores de extrativos, conforme mostrado no Quadro 1.

O teor de extrativos totais apresentou tendência de crescimento em relação à idade e posição do disco ao longo do tronco, no sentido base-topo. O valor médio do teor de extrativos totais foi de $4,08 \%$, enquanto os limites inferior e superior foram, respectivamente, de $2,76 \%$ (idade de 10 anos na posição correspondente à extremidade da segunda tora) e $6,34 \%$ (idade de 20 anos no disco da base), apresentando variação de mais de $297,0 \%$. Os resultados do Quadro 2 ficaram dentro da faixa normalmente encontrada na literatura para Eucalyptus grandis, variando de 2,50 a 7,00\%.

Os valores médios dos teores de extrativos estão apresentados no Quadro 2.

Com tais resultados, verificou-se uma tendência de estabilização dos teores, a partir de certa idade. Observou-se uma diferença estatística entre os teores encontrados nas madeiras de 10, 14 e 25 anos, mas tal diferença não foi constatada entre os teores encontrados nas madeiras de 20 e 25 anos, indicando a tendência de estabilização com a idade. O teor de extrativos da madeira de árvores mais jovens tende a apresentar valores mais reduzidos que os encontrados nas madeiras mais maduras, conforme os resultados da literatura.

\section{2. Teor de lignina total}

Pela análise de variância, verificou-se que os efeitos da idade e da posição do disco ao longo do tronco no teor de lignina total foram significativos em nível de probabilidade de $95 \%$. Também foi verificado que tais efeitos apresentaram a mesma intensidade entre si no teor de lignina; a interação desses efeitos, no entanto, não teve qualquer influência na definição dos valores relacionados aos teores de lignina, não sendo significativo a $95 \%$ de probalidade. Os dados da análise de variância estão apresentados no Quadro 3.

Quadro 2 - Valores médios dos teores de extrativos totais (\%) da madeira de Eucalyptus grandis de diferentes idades e posições ao longo do tronco Table 2 - Average extractive content (\%) along the trunk of Eucalyptus grandis of different ages

\begin{tabular}{|c|c|c|c|c|c|}
\hline Tratamento & Amostras & Médias & Tukey & $\begin{array}{l}\mathrm{CV} \\
(\%)\end{array}$ & $\begin{array}{l}\text { DP } \\
(\%) \\
\end{array}$ \\
\hline Idade 10 anos & 24 & 3,41 & $a^{1 /}$ & 14,78 & 0,505 \\
\hline Idade 14 anos & 24 & 3,85 & $\mathrm{~b}$ & 19,71 & 0,760 \\
\hline Idade 25 anos & 24 & 4,46 & $\mathrm{c}$ & 25,31 & 1,129 \\
\hline Idade 20 anos & 24 & 4,60 & $\mathrm{c}$ & 24,82 & 1,143 \\
\hline Disco 2 & 32 & 3,46 & a & 9,74 & 0,337 \\
\hline Disco 1 & 32 & 3,68 & a & 14,27 & 0,525 \\
\hline \multirow[t]{2}{*}{ Disco base } & 32 & 5,11 & $\mathrm{~b}$ & 21,45 & 1,096 \\
\hline & \multicolumn{4}{|c|}{ Interação Idade X Disco } & \\
\hline $10 \operatorname{anos} x$ disco 2 & 8 & 3,09 & $\mathrm{a}$ & 9,71 & 0,300 \\
\hline $14 \operatorname{anos} x$ disco 2 & 8 & 3,17 & a & 9,27 & 0,294 \\
\hline 10 anos $x$ disco 1 & 8 & 3,24 & $a b$ & 8,95 & 0,290 \\
\hline 20 anos $x$ disco 2 & 8 & 3,65 & $\mathrm{ab}$ & 3,56 & 0,130 \\
\hline $25 \operatorname{anos} x$ disco 2 & 8 & 3,72 & $a b$ & 6,69 & 0,249 \\
\hline $14 \operatorname{anos} x$ disco 1 & 8 & 3,75 & $\mathrm{bc}$ & 13,72 & 0,516 \\
\hline 25 anos $x$ disco 1 & 8 & 3,76 & $\mathrm{bc}$ & 2,18 & 0,082 \\
\hline 10 anos $\mathrm{x}$ disco base & 8 & 3,90 & $\mathrm{~cd}$ & 12,49 & 0,487 \\
\hline $20 \operatorname{anos} x$ disco 1 & 8 & 4,15 & $\mathrm{~cd}$ & 9,18 & 0,381 \\
\hline 14 anos $x$ disco base & 8 & 4,63 & de & 11,90 & 0,551 \\
\hline 25 anos $x$ disco base & 8 & 5,88 & $\mathrm{f}$ & 10,49 & 0,617 \\
\hline 20 anos $x$ disco base & 8 & 6,02 & $\mathrm{f}$ & 12,52 & 0,754 \\
\hline \multicolumn{3}{|l|}{ Média Geral } & \multicolumn{2}{|l|}{4,08} & \\
\hline
\end{tabular}

Quadro 1 - Análise de variância do teor de extrativos da madeira de Eucalyptus grandis, em função da variação da idade e da posição ao longo do tronco

Table 1 - Variance analysis of extractive content (\%) along the trunk of Eucalyptus grandis of different ages

\begin{tabular}{lcccc}
\hline Causa da Variação & G. L. & Soma dos Quadrados & Quadrado Médio & F \\
\hline Principais Efeitos & & & & \\
A: Idade & 3 & 21,9883 & 7,32943 & $34,67 *$ \\
B: Posição do Disco & 2 & 51,2 & 25,6 & $121,09 *$ \\
Interações & 6 & & & 0,0000 \\
AB & 84 & 9,53998 & 1,58998 & $7,52 *$ \\
Resíduo & 95 & 17,7587 & 0,211413 & 0,0000 \\
\hline Total (Corrigido) & 100,487 & & \\
\hline
\end{tabular}

* Significativo A 95\% de probabilidade. 
Quadro 3 - Análise de variância do teor de lignina da madeira de Eucalyptus grandis, em função da variação da idade e da posição ao longo do tronco

Table 3 - Variance analysis of lignin content (\%) along the trunk of Eucalyptus grandis of different ages

\begin{tabular}{|c|c|c|c|c|c|}
\hline Causa da Variação & G. L. & Soma dos Quadrados & Quadrado Médio & $\mathrm{F}$ & $\mathrm{P}$ \\
\hline \multicolumn{6}{|l|}{ Principais Efeitos } \\
\hline A: Idade & 3 & 108,63 & 36,2101 & $20,29 *$ & 0,0000 \\
\hline B: Posição do disco & 2 & 85,4452 & 42,7226 & $23,94 *$ & 0,0000 \\
\hline \multicolumn{6}{|l|}{ Interações } \\
\hline $\mathrm{AB}$ & 6 & 3,33563 & 0,555938 & $0,31 \mathrm{NS}$ & 0,9293 \\
\hline$\underline{\text { Resíduo }}$ & 84 & 149,904 & 1,78457 & & \\
\hline Total (Corrigido) & 95 & 347,315 & & & \\
\hline
\end{tabular}

* Significativo a $95 \%$ de probabilidade e NS não-significativo.

O teor de lignina total apresentou tendência crescente em relação à idade e à posição longitudinal, sentido base-topo. Os resultados do presente estudo estão em consonância com os normalmente encontrados na literatura. O valor médio do teor de lignina total foi de $27,22 \%$, sendo os limites inferior e superior, respectivamente, de $23,78 \%$ (idade de 10 anos no disco da posição 2) e 30,70\% (idade de 20 anos no disco da base), apresentando variação de mais de 29,0\%. Considerando os valores apresentados no Quadro 4, verificou-se a maior variação dos valores $(6,23 \%)$ entre as madeiras de 10 e 14 anos, seguida da variação de $3,06 \%$ entre as madeiras de 14 e 25 anos; entre as madeiras de 20 e 25 anos, a variação foi de apenas 1,21\%. Diversos autores confirmaram, em seus trabalhos, um aumento sistemático do teor de lignina total da madeira com a idade, havendo uma tendência de estabilização, após certa idade da árvore. Os valores médios dos teores de lignina total estão apresentados no Quadro 4.

Quanto à posição no disco, observaram-se diferenças estatísticas entre os valores encontrados nos discos 1 e 2, mas tais diferenças não foram observadas nos discos 1 e da base. O teor de lignina total da madeira de árvores mais jovens tendeu a apresentar valores mais reduzidos que os encontrados nas madeiras mais maduras.

\section{3. Teor de holocelulose}

Pela análise de variância, verificou-se que os efeitos da idade e da posição do disco ao longo do tronco no teor de holocelulose foram significativos em nível de probabilidade de $95 \%$. Também foi verificado que o efeito da posição do disco ao longo do tronco foi muito mais pronunciado que a idade; a interação desses efeitos não teve qualquer influência na definição dos valores relacionados aos teores de holocelulose, não sendo significativa em nível de $95 \%$ de probabilidade. Os valores da análise de variância estão apresentados no Quadro 5.

Quadro 4 - Valores médios dos teores de lignina total (\%) da madeira de Eucalyptus grandis de diferentes idades e posições ao longo do tronco

Table 4-Average lignin content (\%) of disks taken at different positions along the trunk of Eucalyptus grandis, of different ages

\begin{tabular}{lccccc}
\hline Tratamento & Amostras Médias Tukey & $\begin{array}{c}\text { CV } \\
(\%)\end{array}$ & $\begin{array}{c}\text { DP } \\
(\%)\end{array}$ \\
\hline Idade 10 anos & 24 & 25,53 & $\mathrm{a}^{1}$ & 5,58 & 1,426 \\
Idade 14 anos & 24 & 27,12 & $\mathrm{~b}$ & 6,22 & 1,686 \\
Idade 25 anos & 24 & 27,95 & bc & 5,33 & 1,491 \\
Idade 20 anos & 24 & 28,29 & $\mathrm{c}$ & 6,40 & 1,811 \\
Disco 2 & 32 & 26,41 & a & 6,34 & 1,675 \\
Disco 1 & 32 & 26,71 & a & 5,79 & 1,546 \\
Disco base & 32 & 28,59 & b & 6,31 & 1,803 \\
\hline \multicolumn{7}{c}{ Interação Idade X Disco } \\
\hline 10 anos x disco 2 & 8 & 24,73 & a & 7,27 & 1,798 \\
10 anos x disco 1 & 8 & 25,35 & ab & 4,11 & 1,041 \\
14 anos x disco 1 & 8 & 26,35 & ab & 6,20 & 1,634 \\
14 anos x disco 2 & 8 & 26,40 & abc & 5,86 & 1,547 \\
10 anos x disco base & 8 & 26,53 & abc & 2,55 & 0,676 \\
25 anos x disco 2 & 8 & 27,01 & bc & 2,24 & 0,606 \\
20 anos x disco 2 & 8 & 27,51 & bcd & 4,27 & 1,176 \\
25 anos x disco 1 & 8 & 27,56 & bcde & 2,80 & 0,773 \\
20 anos x disco 1 & 8 & 27,59 & bcde & 5,46 & 1,507 \\
14 anos x disco 1 & 8 & 28,63 & cde & 2,28 & 0,650 \\
25 anos x disco base & 8 & 29,23 & de & 6,07 & 1,776 \\
20 anos x disco base & 8 & 29,76 & e & 6,18 & 1,840 \\
\hline Média Geral & \multicolumn{5}{c}{27,22} \\
\hline
\end{tabular}

${ }^{1 /}$ Médias seguidas da mesma letra não diferem estatisticamente, pelo teste de Tukey a $5 \%(\mathrm{p}<0,05)$ de significância. 
Quadro 5 - Análise de variância do teor de holocelulose da madeira de Eucalyptus grandis, em função da variação da idade e da posição ao longo do tronco

Table 5 - Variance analysis of hollocelulose content (\%) along the trunk of Eucalyptus grandis of different ages

\begin{tabular}{|c|c|c|c|c|c|}
\hline Causa da Variação & G. L. & Soma dos Quadrados & Quadrado Médio & $\mathrm{F}$ & $\mathrm{P}$ \\
\hline \multicolumn{6}{|l|}{ Principais Efeitos } \\
\hline A: Idade & 3 & 192,244 & 64,0813 & $31,25^{*}$ & 0,0000 \\
\hline B: Posição do disco & 2 & 85,4452 & 42,7226 & $23,94 *$ & 0,0000 \\
\hline \multicolumn{6}{|l|}{ Interações } \\
\hline $\mathrm{AB}$ & 6 & 3,33563 & 0,555938 & $0,31 \mathrm{NS}$ & 0,9293 \\
\hline$\underline{\text { Resíduo }}$ & 84 & 149,904 & 1,78457 & & \\
\hline Total (Corrigido) & 95 & 347,315 & & & \\
\hline
\end{tabular}

* Significativo a 95\% de probabilidade e NS Não-significativo.

O teor de holocelulose apresentou tendência inversamente proporcional em relação à idade. $\mathrm{O}$ valor médio do teor de holocelulose foi de $68,69 \%$, sendo os limites inferior e superior, respectivamente, de $63,21 \%$ (idade de 20 anos no disco da base) e 73,11\% (idade de 14 anos no disco da posição 2), apresentando variação de mais de $15,66 \%$. Os valores médios encontram-se no Quadro 6.

O teor de holocelulose é maior nas árvores mais jovens que os encontrados nas madeiras mais maduras. Embora haja controvérsias, diversos autores confirmaram a diminuição sistemática do teor de holocelulose da madeira com o aumento da idade, havendo tendência de estabilização após certa idade da árvore. Os resultados do presente estudo para o teor de celulose estão em consonância com os normalmente encontrados na literatura. Estatisticamente, os discos nas posições 1 e 2 não apresentaram diferenças significativas entre si, mas diferiram significativamente do disco da base; entre as idades de 10, 14 e 25 anos, observaram-se diferenças significativas entre si, as quais, porém, não foram encontradas nas idades de 20 e 25 anos.

\section{CONCLUSÕES}

Com base nos resultados, pode-se concluir que:

a) A composição química da madeira foi influenciada pela idade e pela variação da posição do disco no sentido longitudinal.

b) A variabilidade interna das árvores no sentido longitudinal influiu mais decisivamente na composição química da madeira que a variação da idade.

c) As toras da base das árvores e de maior idade apresentaram maiores teores de extrativos e lignina que as demais toras, correspondendo a madeiras mais maduras.

e) A madeira das árvores mais jovens e localizada nas partes mais superiores do tronco apresentaram maiores teores de holocelulose.

Quadro 6 - Valores médios dos teores de holocelulose (\%) da madeira de Eucalyptus grandis, de diferentes idades (anos) e posições ao longo do tronco

Table 6-Average holocellulose content (\%) of disks taken at different positions along the trunk of Eucalyptus grandis, of different ages

\begin{tabular}{lccccc}
\hline Tratamento & Amostras Médias Tukey & $\begin{array}{c}\text { CV } \\
(\%)\end{array}$ & $\begin{array}{r}\text { DP } \\
(\%)\end{array}$ \\
\hline Idade 20 anos & 24 & 67,11 & a $^{1}$ & 3,74 & 2,513 \\
Idade 25 anos & 24 & 67,59 & a & 3,72 & 2,517 \\
Idade 10 anos & 24 & 69,46 & b & 2,95 & 2,050 \\
Idade 14 anos & 24 & 70,61 & c & 2,44 & 1,726 \\
Disco base & 32 & 66,34 & a & 3,72 & 2,471 \\
Disco 1 & 32 & 69,61 & b & 2,45 & 1,705 \\
Disco 2 & 32 & 70,13 & b & 2,56 & 1,796 \\
\hline \multicolumn{7}{c}{ Interação Idade X Disco } \\
\hline 20 anos x disco base & 8 & 64,22 & a & 2,68 & 1,720 \\
25 anos x disco base & 8 & 64,85 & a & 3,93 & 2,550 \\
14 anos x disco base & 8 & 67,47 & b & 1,61 & 1,088 \\
20 anos x disco 1 & 8 & 68,26 & bc & 2,09 & 1,429 \\
25 anos x disco 1 & 8 & 68,71 & bcd & 1,38 & 0,950 \\
10 anos x disco base & 8 & 68,84 & bcd & 0,42 & 0,288 \\
20 anos x disco 2 & 8 & 68,85 & bcd & 3,70 & 2,550 \\
25 anos x disco 2 & 8 & 69,21 & bcd & 0,89 & 0,615 \\
14 anos x disco 1 & 8 & 70,35 & cde & 2,40 & 1,687 \\
14 anos x disco 2 & 8 & 70,59 & cde & 2,44 & 1,722 \\
10 anos x disco 1 & 8 & 70,89 & de & 1,47 & 1,040 \\
10 anos x disco 2 & 8 & 72,11 & e & 2,15 & 1,548 \\
\hline Média Geral & \multicolumn{5}{c}{68,69} \\
\hline \hline
\end{tabular}

${ }^{1 /}$ Médias seguidas da mesma letra não diferem estatisticamente, pelo teste de Tukey com significância de $5 \%(\mathrm{p}<0,05)$.

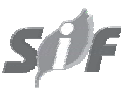

R. Árvore, Viçosa-MG, v.29, n.3, p.455-460, 2005 


\section{REFERÊNCIAS BIBLIOGRÁFICAS}

CHAFE, S.C. Relationships between shrinkage and specific gravity in the wood of Eucalyptus.

Australian Forestry, v. 57, p. 59-61, 1994.

GOLDSCHIMID, O. Ultraviolet spectra. In: SARKANEN, K. V.; LUDWIG, C. H. Lignins: occurrence, formation, structure and reactions. New York: John Wiley Interprice, 1971. p. 241-298.

GOMIDE, J. L. ; DEMUNER, B. J. Determinação do teor de lignina em material lenhoso: método klason modificado. O Papel, v. 47, n. 8, p. 3638. 1986.

GONÇALEZ, J. C. Caracterization technologique de quatre espèces peu connues de la Forêt Amazonienne: anatomie, chimie, couleur, proprietés physiques et mécaniques. 1993. 444f. Thèse (Doctorat- Technologie du Bois) École Nationale du Gêne Rural des Eaux et des Forêts. Nancy, 1993.

HILLIS, W. E.; BROWN, A. G. Eucalyptus for wood production. Melbourne: CSIRO, 1978. $434 p$.

JANKOWSKY, I. P. Influência da densidade básica e do teor de extrativos na umidade de equilíbrio da madeira. 1979. 87f. Dissertação (Mestrado em Engenharia Florestal), Escola Superior de Agricultura “Luiz de Queiroz", Universidade de São Paulo, Piracicaba, 1979.

KRAMER, P.J.; KOZLOWSKI, T. T. Physiology of woody plants. New York: Academic Press, 1979. 745p.
LEPAGE, E. S. Química da Madeira. In: MANUAL de preservação de madeiras, São Paulo: IPT, 1986. p. 69-97

PANSHIN, A . J.; DE ZEEUW, C. Textbook of wood technology. 4. ed. New York: McGraw Hill, 1980. 722p.

RAYMOND, C. A . Tree breeding issues for solid wood products. In: THE FUTURE OF EUCALYPTS FOR WOOD PRODUCTS. 2000, Launceston,

Tasmania. Proceedings.... Launceston: IUFRO, 2000, p. $265-270$.

SANTOS, F.L.C. Perspectivas do mercado nacional de madeiras reflorestadas oriundas de florestas plantadas. In: SIMPÓSIO IPEF - A

REENGENHARIA E SEUS IMPACTOS NO

DESENVOLVIMENTO CIENTIIFICOE

TECNOLÓGICO DO SETOR FLORESTAL, 4., 1996 , São Paulo. Anais ... São Pedro: IPEF, 1996. v.3, p. 31-52.

SILVA, J. C. Caracterização da madeira de Eucalyptus grandis Hill ex Maiden, de diferentes idades, visando a sua utilização na indústria moveleira. 2002. 160f. Tese (Doutorado em Engenharia Florestal) Universidade Federal do Paraná, Curitiba, 2002.

SILVA, J. R. M. Relações da variabiidade e aderência do verniz com as propriedades fundamentais do Eucalyptus grandis Hill ex. Maiden. 2002. 179 f. Tese (Doutorado em Engenharia Florestal) - Universidade Federal do Paraná, Curitiba, 2002.

ZOBEL, J.B.; BUJTENEN, J.P. Wood variation: its causes and control. New York: Springer-Verlag, 1989. 363p. 